\title{
aPKC-mediated persistent increase in AMPA/NMDA ratio in the VTA participates in the neuroadaptive signal necessary to induce NAc synaptic plasticity after cocaine administration
}

\author{
Ana del C. Vaquer-Alicea, B.S. ${ }^{1}$, Rafael Vázquez Torres, B.S ${ }^{1}$, Marcos Devarie-Hornedo, \\ M.D. ${ }^{2}$, Juan C. Vicenty-Padilla, M.D. ${ }^{3}$, Bermary Santos Vera, Ph.D. ${ }^{1}$, Cristina María-Ríos, \\ B.S. ${ }^{4}$, Maria E. Vélez-Hernández, Ph.D. ${ }^{5}$, Todd Sacktor, M.D. ${ }^{6}$, and Carlos A. Jiménez- \\ Rivera, Ph.D. ${ }^{1}$ \\ ${ }^{1}$ Department of Physiology, University of Puerto Rico Medical Sciences Campus, San Juan, P.R. \\ ${ }^{2}$ School of Medicine, University of Puerto Rico Medical Sciences Campus, San Juan, P.R. \\ ${ }^{3}$ Department of Neurosurgery, University of Puerto Rico Medical Sciences Campus, San Juan, \\ P.R. \\ ${ }^{4}$ Department of Biology, University of Puerto Rico Rio Piedras Campus, San Juan, P.R. \\ ${ }^{5}$ Department of Biological and Health Sciences, University of Houston-Victoria, Houston, Texas, \\ U.S.A. \\ ${ }^{6}$ Department of Physiology and Pharmacology, The Robert F. Furchgott Center for Neural and \\ Behavioral Science, State University of New York Downstate Medical Center, Brooklyn, NY \\ 11203, U.S.A.
}

\section{Abstract}

Chronic cocaine exposure produces enduring neuroadaptations in the brain's reward system.

Persistence of early cocaine evoked neuroadaptations in the ventral tegmental area (VTA) are necessary for later synaptic alterations in the nucleus accumbens (NAc), suggesting a temporal sequence of neuroplastic changes between these two areas. However, the molecular nature of the signal that mediates this sequential event is unknown. Here we used the behavioral sensitization model and the aPKC inhibitor of late-phase LTP maintenance, ZIP, to investigate if a persistent increase in AMPA/NMDA ratio plays a role in the molecular mechanism that allows VTA neuroadaptations to induce changes in the NAc. Results showed that intra-VTA ZIP microinfusion successfully blocked cocaine evoked synaptic enhancement in the VTA and the expected AMPA/ NMDA ratio decrease in the NAc following cocaine sensitization. ZIP microinfusions also blocked the expected AMPA/NMDA ratio increase in the NAc following cocaine withdrawal. These results suggest that a persistent increase in AMPA/NMDA ratio, mediated by aPKC's, could be the

Correspondence: Carlos A. Jiménez-Rivera, Ph.D. University of Puerto Rico Medical Sciences Campus, Américo Miranda Ave., Main Building, Department of Physiology, 6th floor, Room A688, San Juan, Puerto Rico 00936, Tel: 787-758-2525 ×1677, carlos.jimenez8@upr.edu.

Publisher's Disclaimer: This is a PDF file of an unedited manuscript that has been accepted for publication. As a service to our customers we are providing this early version of the manuscript. The manuscript will undergo copyediting, typesetting, and review of the resulting proof before it is published in its final citable form. Please note that during the production process errors may be discovered which could affect the content, and all legal disclaimers that apply to the journal pertain. 
molecular signal that enables the VTA to elicit synaptic alterations in the NAc following cocaine administration.

\section{Keywords}

VTA plasticity; NAc plasticity; PKM- $\zeta$; ZIP; Sprague Dawley

\section{Introduction}

Drug addiction is a chronic relapsing disease characterized by enduring alterations in the mesocorticolimbic system's response to rewarding stimuli. These changes drive the persistent habit-learning behavior associated with compulsive drug use (Everitt and Wolf, 2002; Hyman et al, 2006). Drugs of abuse induce an increase in dopaminergic and glutamatergic neurotransmission that facilitates neuronal excitability (Di Chiara and Imperato, 1988; Saal et al, 2003; Tan et al, 2010). Furthermore, drug exposure leads to the formation of long-term potentiation (LTP) (Hyman et al, 2006). LTP is considered the cellular substrate for memory formation (Bliss and Lomo, 1973; Lynch, 2004), and a facilitator of the protractive nature of the addictive process (Ungless et al, 2001; Saal et al, 2003).

Cocaine can evoke an increase in synaptic strength throughout the mesocorticolimbic system, yet the functional significance of this event is not well understood. Several studies suggest that the enhanced plasticity in the ventral tegmental area (VTA) is a temporary event that participates in the early changes that occur in the reward circuit ( Ungless et al, 2001;Boudreau and Wolf, 2005; Boudreau et al, 2007;). In contrast, the NAc develops persistent synaptic enhancement at a later time-point (Kourrich et al, 2007). The chronological sequence of synaptic potentiation between these two structures suggests that initial changes in the VTA promote later alterations in the NAc. Recently, it was shown that synaptic potentiation in the VTA is crucial for NAc neuroadaptions to occur (Mameli et al, 2009). However, the cellular mechanisms responsible for this hierarchical organization remain to be elucidated.

In vitro experiments demonstrate that the maintenance of cocaine-evoked synaptic enhancement in the VTA is susceptible to the myristoylated atypical PKC pseudosubstrate inhibitory peptide, ZIP (Ho et al, 2012; Vélez-Hernández et al, 2013), which specifically affects the maintenance of the late-phase LTP (Ling et al, 2002) by stimulating the autoinhibitory regulatory subunit of atypical PKCs (Pastalkova et al, 2006). Behavioral studies in cocaine-exposed rats revealed that cocaine exposure increases PKM- $\zeta$ in the VTA (Ho et al, 2012). In addition, ZIP microinfusions in the VTA and NAc disrupt cocainerelated behaviors (Li et al, 2011; Shabashov et al, 2011; Vélez-Hernández et al, 2013). Whether this disruption is long-lasting (Shema et al, 2007) or can be naturally regenerated by reward circuit redundancy (Parsons and Davis, 2011), is still contested.

Knowing cocaine evoked behaviors rely on VTA and NAc synaptic potentiation, aPKCmediated synaptic enhancement could be part of the molecular mechanisms that mediates the sequential generation of plasticity between these two areas. In the present 
study, we used the cocaine behavioral sensitization model to investigate the molecular mechanisms that allows for a hierarchical order of cocaine-elicited plasticities between the VTA and NAc. The permanency of these effects was also tested. We hypothesize that persistent increase in AMPA/NMDA ratio in the VTA is one of the cellular events responsible for the neuroadaptations in the NAc.

\section{Experimental Procedures}

\section{Animal Housing}

Sprague Dawley male rats (Taconic farms, Germantown, N.Y.) were housed in pairs in cages located at the Animal Resource Center (ARC) of our institution. They were exposed to $12 \mathrm{hr}$ light/dark cycles. Water and food were available ad libitum. Surgical procedures and ZIP microinfusions took place in the ARC, while behavioral experiments were done in our laboratory. All animal procedure protocols were approved by our institutional IACUC committee.

\section{Bilateral guide cannula placement for intracranial ZIP microinjections}

Sprague Dawley male rats (200-230g) were anesthetized with pentobarbital $(50 \mathrm{mg} / \mathrm{kg})$, administered atropine $(0.1 \mathrm{mg} / \mathrm{kg})$ and Metacam $(1 \mathrm{mg} / \mathrm{kg})$. A bilateral cannula (Plastics One, Roanoke, VA) was placed using a stereotaxic apparatus (Kopf Instruments, Tujunga, CA). Stereotaxic coordinates (Paxinos and Watson atlas, 1998) for the VTA were: AP= $-5.3 \mathrm{~mm}$ caudal from bregma, and $-7.0 \mathrm{~mm}$ ventral from cortical surface. For the NAc: $+2.3 \mathrm{~mm}$ rostral from bregma, and $-5.2 \mathrm{~mm}$ ventral from cortical surface. After cannula placement (1 $\mathrm{mm}$ above the desired area) the incision was sutured, and a topical antibiotic was applied to prevent infection. Animals were allowed to recover for 7 days.

\section{Cocaine behavioral sensitization}

Cocaine behavioral sensitization is defined as a progressive increase in locomotion following consecutive drug administrations, which persists after an abstinence period (Kalivas and Duffy, 1993). Behavioral procedures were performed as previously described (VélezHernández et al, 2013). Animals were randomly assigned to 4 groups (Vehicle/Saline, ZIP/ Saline, Vehicle/Cocaine, and ZIP/Cocaine) and subjected to the cocaine sensitization paradigm. Locomotion was assessed inside an isolated acoustic chamber (Whisper Room Inc., Morristown, TN, USA). Two days before the beginning of the experiment, each group was habituated for 1 hour to the infrared photocell box (Accuscan Instruments, Columbus, $\mathrm{OH}, \mathrm{USA}$ ). Locomotion units were assessed as photocell counts (pcc). On experimental day 1 through 5, animals were habituated for 15 minutes and were then treated with either 15 $\mathrm{mg} / \mathrm{kg}$ i.p. Cocaine (Sigma, St. Louis, MO, USA) or isovolumetric saline injections. Immediately after the injections, locomotion activity was assessed for one hour. On challenge day, all animals received Cocaine. Total locomotion was measured as the total number of times the rat interrupted an infrared beam. Interruption of consecutive beams was considered as ambulatory locomotion. Consecutive interruption of the same beam was counted as stereotypic locomotion. 
Daily VTA ZIP or vehicle microinfusions were performed with a microinfusion pump (ColePalmer, Chicago IL), six hours after cocaine injection. Vehicle/saline and Vehicle/Cocaine groups received vehicle through a cannula and either saline or cocaine injections (i.p.). ZIP/ Saline and ZIP/Cocaine received ZIP through the cannula and either saline or cocaine injections (i.p.), respectively. NAc ZIP microinfused rats were divided into the same groups and sensitized as explained above. They were allowed a 7day withdrawal period followed by bilateral NAc ZIP microinfusions, and allowed another 7day withdrawal period before getting a cocaine challenge.

Averaged data is presented as mean \pm SEM and analyzed using Two-way repeated measures ANOVA followed by Sidak's and Tukey's post-hoc test. A p $<0.05$ between day 5 and day 1 of the sensitization protocol is considered a successful sensitization (Kalivas and Stewart, 1991).

\section{Drug Infusion}

ZIP (Myr-SIYRRGARRGARRWRKL-OH, Anaspec, CA) was dissolved in a $100 \mathrm{mM}$ Trissaline solution ( $\mathrm{pH}$ 7.3). Rats were microinfused bilaterally with $10 \mathrm{nmol} \mathrm{ZIP}(0.5 \mu \mathrm{l} / \mathrm{side}$, $0.1 \mathrm{ul} / \mathrm{min}$ ) using a $10 \mu \mathrm{l}$ syringe (Hamilton, Reno, NV). The bilateral cannula was left in place for an additional minute to avoid drug backflow.

\section{Electrophysiological studies}

Rats were sacrificed at different time points of the behavioral experiments: on day 5, after a 7-day withdrawal period, and 24 hours following a cocaine challenge. Animals were anesthetized with chloral hydrate $(400 \mathrm{mg} / \mathrm{Kg})$ and decapitated. Brains were removed and placed in cold $\left(2-4^{\circ} \mathrm{C}\right)$ and oxygenated $\left(95 \% \mathrm{O}_{2}\right.$ and $5 \% \mathrm{CO}_{2}$ gas mixture to $\left.\mathrm{pH}=7.4\right)$ artificial cerebrospinal fluid (ACSF) containing (in $\mathrm{mM}$ ): $127 \mathrm{NaCl} ; 2.5 \mathrm{KCl} ; 1.25$ $\mathrm{NaH}_{2} \mathrm{PO}_{4} ; 25 \mathrm{NaHCO}_{3} ; 2 \mathrm{CaCl}_{2} ; 1 \mathrm{MgCl}_{2} ; 25$ D-glucose. NAc coronal and horizontal VTA sections were taken using a Vibratome (VT1000S, Leica, Germany). Slices were transferred to an incubation chamber containing oxygenated ACSF at $32^{\circ} \mathrm{C}$ for $1 \mathrm{hr}$ and subsequently placed in a recording chamber superfused with oxygenated $\operatorname{ACSF}\left(25^{\circ} \mathrm{F}\right)$ at $2 \mathrm{~mL} / \mathrm{min}$. ACSF was supplemented with Picrotoxin $(100 \mu \mathrm{M})$ to block $\mathrm{GABA}_{\mathrm{a}}$ receptor mediated IPSC's. Whole-cell voltage clamp recordings were obtained from putative dopaminergic (DA) cells of the VTA and medium spiny neurons (MSN) of the NAc core. They were identified as previously described (Velez-Hernandez et al, 2013; Velazquez-Martinez et al, 2015), using infrared microscopy with DIC (BX51WI Olympus, Japan). Recordings from putative VTA DA were done in the region lateral to the fasciculus retroflexus and medial to the medial terminal nucleus of the accessory optic tract. Recordings from putative NAc (MSN) were done in the region lateral to the NAc shell and medial to the anterior commissure (Paxinos and Watson, 1998). The NAc core was chosen for these experiments since it has been demonstrated that it contributes to drug associated, cue-induced cocaine seeking and to the incentive value of reward-conditioned stimuli (Hollander and Carelli, 2007; Goto and Grace, 2008; Owesson-White et al, 2009). Moreover, the NAc core undergoes synaptic strengthening after repeated exposure to cocaine followed by a withdrawal period (Korrich et al, 2007; Ferrario et al, 2010). Recent studies suggest that the NAc core is the key structure where DA release attributes incentive salience and 
motivational properties to reward cues which are of the utmost importance in the cocaine sensitization model used here (Saunders and Robinson, 2012). These findings have been currently supported using optogenetic techniques that investigated DA circuit-specific functions in Pavlovian reward learning (Saunders et al, 2018).

Borosilicate glass patch pipettes were pulled to a final resistance of 3-6 $\mathrm{M} \Omega$ and filled with (in $\mathrm{mM}$ ): 120 cesium methanesulfonate, 20 HEPES, 0.4 EGTA, $2.8 \mathrm{NaCl}, 5 \mathrm{TEA}-\mathrm{Cl}, 2.5$ MgATP and 0.25 NaGTP, pH 7.2-7.3 (270-285 mOsm). A bipolar stimulating electrode was placed 50-150 $\mu \mathrm{M}$ rostral to the recording electrode to stimulate at $100 \mathrm{~ms}$ intervals. AMPA/ NMDA ratios were measured as described by Thomas et al, 2001. Briefly, cells were clamped at $+40 \mathrm{mV}$ and the NMDAR inhibitor D-2-amino-5phosphonovalerate (D-APV) $(50 \mu \mathrm{M})$ was applied to the superfusion medium. NMDAR responses were calculated by

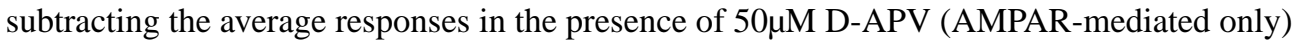
from that recorded in its absence. Experiments with a series resistance change of more than $20 \%$ were discarded. Averaged data are presented as a mean \pm SEM and analyzed using Two-way ANOVA followed by Sidak's post-hoc test.

Data was collected with PClamp 9 software through a Multiclamp 700B amplifier (Molecular Devices, Sunnyvale, Ca), filtered at $10 \mathrm{kHz}$ through a Bessel filter, digitized at 5 kHz using Digidata 1322A (Axon Instruments, Foster City, CA), and analyzed off line using Clampfit (Molecular Devices) software.

\section{Histological verification of cannula placement}

Following behavioral experiments, animals were anesthetized with $50 \mathrm{mg} / \mathrm{kg}$ of pentobarbital and the microinfusion site was marked with Pontamine Sky Blue (0.5ul/side). Cannula placement was determined using microscopic examination of Pontamine Sky Blue in brain sections. Animals that received microinfusions outside of the region of interest were excluded from data analyses.

Statistical analyses and figures were done using Graph Pad PRISM software.

\section{Results:}

\section{Daily ZIP microinjections into the VTA alter AMPA/NMDA ratios in the VTA and NAC}

ZIP inhibition of aPKC's has been shown to disrupt AMPA receptor trafficking to the membrane, decreasing AMPA/NMDA ratios. AMPA/NMDA ratios are an electrophysiological signature of LTP expression (Lu et al, 2001). As a normalization procedure, it allows the comparison of the strength of synaptic potentiation of excitatory synapses between slices, by correcting for electrode placement and density of fibers activated. Recording electrode and stimulating electrode placement illustrated in Fig. 1C and Fig. 1F represent the configurations used in all AMPA/NMDA ratio experiments (Fig. 1, 2, and 3).

First, we assessed if in vivo ZIP microinfusions in the VTA could effectively block AMPA/ NMDA ratio increase following a single cocaine exposure (Fig. 1A). Additionally, we examined AMPA/NMDA ratios in MSN of the NAc core to see if this disruption had any 
effect on NAc synaptic plasticity. Twenty-four hours following a responsive single cocaine injection (ANOVA $F(1,28)=0.1239, p=0.72$, Tukey's multiple comparisons test significant $\mathrm{p}=0.001$ (Vehicle/Saline vs Vehicle/Cocaine), $\mathrm{p}=0.001$ (Vehicle /Saline vs ZIP/Cocaine), $\mathrm{p}=0.001$ (ZIP/Saline vs Vehicle/Cocaine), and $\mathrm{p}=0.001$ (ZIP/Saline vs ZIP/Cocaine) $)($ Fig 1.B) and a ZIP microinfusion, there is a statistical significant difference in AMPA/NMDA ratios in VTA DA neurons between Vehicle/Cocaine and ZIP/Cocaine groups (ANOVA F $(1,22)=$ 21.72, $\mathrm{p}=0.0001)$. The increased AMPA/NMDA ratio of Vehicle/Cocaine animals (1.12 \pm 0.06 ) is not observed in ZIP/Cocaine rats $(0.47 \pm 0.02)$ (Fig. 1E). In the NAc core, there was no statistical significant difference between the NAc AMPA/NMDA ratios of Vehicle/ Cocaine group and ZIP/Cocaine group (Fig. 1H). This reveals that AMPA/NMDA ratio enhancement in the VTA can be effectively blocked after a single microinfusion.

Next, we set out to explore if aPKC-mediated persistent increase in AMPA/NMDA ratio is the molecular mechanism underlying the hierarchical nature of plasticity between the VTA and the NAc. For this, we made daily intra-VTA ZIP microinfusions during the initiation of cocaine behavioral sensitization and evaluated AMPA/NMDA ratios in the VTA at different time-points of the behavioral sensitization model: on day 5 of daily cocaine and zip microinfusions, following a 7-day cocaine withdrawal, and 24 hours after a cocaine challenge. This last timepoint would allow us to see if ZIP disruption of plasticity in the VTA would preclude the formation of new cocaine-evoked synaptic potentiation. We also measured AMPA/NMDA ratios in the MSN of the NAc core to reveal if disruption of VTA AMPA/NMDA ratio had an effect in NAc synaptic plasticity.

After 5 days of cocaine injections and ZIP microinfusions (Fig. 2A), there is a statistically significant difference in AMPA/NMDA ratios in VTA DA neurons between Vehicle/Cocaine and ZIP/Cocaine groups (ANOVA $F(1,22)=20.88, \mathrm{p}=0.0002$ ). The increased AMPA/NMDA ratio of Vehicle/Cocaine animals (1.24 \pm 0.1$)$ is not observed in ZIP/Cocaine animals $(0.53$ \pm 0.02 ) (Fig. 2D). In the NAc core, Vehicle/Cocaine animals showed a statistically significant decrease in AMPA/NMDA ratio $(0.99 \pm 0.06)$, which was not present in ZIP/ Cocaine animals $(1.37 \pm 0.07$ ) (ANOVA $F(1,44)=4.585$, $\mathrm{p}=0.0378$ ) (Fig. $2 \mathrm{G}$ ). Therefore, intra-VTA ZIP microinfusions blocked the decrease in AMPA/NMDA ratio in the NAc after consecutive cocaine injections.

Following a 7-day withdrawal period (Fig. 3A), VTA DA cells of Vehicle/Cocaine animals continued to exhibit a statistically significant increased AMPA/NMDA ratio $(1.22 \pm 0.06)$ when compared with ZIP/Cocaine animals $(0.55 \pm 0.015)$ (ANOVA $F(1,24)=87.33$, $\mathrm{p}<0.0001$ ) (Fig. 3C). NAc core MSN revealed an increase AMPA/NMDA ratio in Vehicle/ Cocaine animals $(1.61 \pm 0.07)$ when compared with ZIP/Cocaine animals $(0.1 .32 \pm 0.04)$ (ANOVA $F(1,33)=6.526, p=0.0154)$ (Fig. 3E). This implies daily intra-VTA ZIP microinfusions also blocked an increase in AMPA/NMDA ratio in the NAc core following a cocaine withdrawal period.

Finally, 24 hours after a cocaine challenge (Fig. 4A), there was no statistical significant difference between the VTA and NAc AMPA/NMDA ratios of Vehicle/Cocaine group and ZIP/Cocaine group (Fig. 4C, E). 


\section{Daily ZIP microinjections into the VTA attenuate initiation but not the expression of cocaine sensitization}

Previously, our laboratory showed that a single ZIP microinjection in the VTA on day 5 of a cocaine behavioral sensitization protocol blocked initiation of sensitization but not the expression (Velez-Hernandez et al, 2013). We hypothesized that uninterrupted neural signaling from the VTA to the NAc for 5 days before inhibiting VTA plasticity with ZIP, triggered the necessary plastic changes in the NAc for the expression of cocaine sensitization. Here we examine if daily interruption of cocaine-induced VTA plasticity can alter cocaine sensitization initiation and expression (Fig. 5A). Rats received cocaine or saline i.p. injections for 5 consecutive days, to induce cocaine sensitization. Six hours after each cocaine injection, they received bilateral intra-VTA ZIP or Vehicle microinfusions (Fig. 5B). To determine ZIP's effects on the initiation of cocaine sensitization, we examined locomotion changes on day 5 of the protocol. Vehicle/Cocaine and ZIP/cocaine rats demonstrated cocaine sensitization on day $5(9738.2 \pm 1036.6$ counts and $6851.5 \pm 930.1$ counts, respectively), compared to day 1 (3722 \pm 356.45 counts and 3,022 \pm 405.56 counts respectively; ANOVA $F(4,124)=1.475, \mathrm{p}=0.2139$, Tukey's multiple comparison test significant $\mathrm{p}=0.0001$ ). Total locomotor sensitization of ZIP/Cocaine animals was significantly reduced on day 5, when compared to Vehicle/Cocaine animals (ANOVA $\mathrm{F}(4,124)=1.475, \mathrm{p}=0.2139$, Sidak's multiple comparison test significant $\mathrm{p}=0.01$ ) (Fig. 5C). Ambulatory movement of Vehicle/Cocaine and ZIP/Cocaine groups were significantly increased by day 5 ( $2857.3 \pm 355.12$ counts for, and $2825.7 \pm 340.95$ counts, respectively), compared to day 1 ( $1908.4 \pm 206.04$ counts and $1544.4 \pm 192.0$ counts respectively; ANOVA $\mathrm{F}(4,124)=0.310, \mathrm{p}=0.87$, Tukey's multiple comparison test significant $\mathrm{p}=0.001$ ). No significant differences were observed between Vehicle/Cocaine and ZIP/Cocaine groups (Fig. 5D). Stereotypic movement of Vehicle/Cocaine and ZIP/Cocaine was significantly increased by day 5 (6202.3 \pm 1186.5 counts, and $4026.3 \pm 646.9$ counts, respectively), compared to day $1(1691.0 \pm 229.1$ counts and $1478.0 \pm 244.5$ counts respectively; ANOVA $\mathrm{F}(4,124)=2.509, \mathrm{p}=0.0453$, Tukey's multiple comparison test significant $\mathrm{p}=0.001) . \mathrm{ZIP} /$ Cocaine group stereotypic movement on day 5 was significantly reduced when compared to the Vehicle/Cocaine group (ANOVA F $(4,124)=2.509, \mathrm{p}=0.0453$, Sidak's multiple comparison test significant $\mathrm{p}=0.001$ ) (Fig. 5E).

To determine if persistent inhibition of VTA cocaine-evoked plasticity blocks the expression of sensitization, animals were allowed a 7-day withdrawal period followed by a cocaine challenge (Fig 5A). On challenge day, ZIP/Cocaine pre-treated animals showed no statistical difference in total, ambulatory or stereotypic locomotion, compared to Vehicle/Cocaine rats (Fig. 5C, F, and I).

\section{Discussion}

How memories are maintained in the brain has been a fundamentally unanswered question in science. Currently, LTP is the most commonly studied physiological model of memory. Inhibition of the late-phase of LTP by ZIP has been shown to erase spatial, instrumental, and classically conditioned long-term memories (Pastalkova et al, 2006; Shema et al, 2007; 2009). Surprisingly, this advance has been scarcely tested for addiction-related plasticities in 
the mesolimbic DA system ( Li et al, 2011; Ho et al, 2012; Vélez-Hernández et al, 2013). Recently it was proposed that the neuroplastic changes between the VTA and the NAc follows a pattern; persistence of VTA plasticity is needed for NAc neuroadaptations (Mameli et al, 2009). In the present study, we explored if the hierarchical nature of this plasticity relies on an aPKC-mediated persistent increase in AMPA/NMDA ratio. We observed that in vivo ZIP administration in the VTA modified in vitro AMPA/NMDA ratios in both the VTA and NAc core at different time points of the behavioral sensitization paradigm.

ZIP is a myristoylated aPKC pseudosubstrate inhibitory peptide that simulates the autoinhibitory regulatory subunit of atypical PKCs (Pastalkova et al, 2006). aPKC phosphorylation enhances AMPA receptor neurotransmission during the late-phase of LTP by increasing and maintaining AMPA receptors in synapses (Ling et al, 2002; Serrano et al, 2005). aPKC inhibition disrupts AMPA receptor incorporation at potentiated synapses and it is associated with retrograde amnesia of long-term memories (Pastalkova et al, 2006; Shema et al, 2009). The identity of the aPKC that mediates these effects has been heavily disputed (Lee et al, 2013; Volk et al, 2013). Recent evidence indicates that ZIP's effect in wild-type mice is likely due to specific inhibition of PKM- $\zeta$, whereas its ability to reverse LTP and erase memory in PKM- $\zeta$-knockout mice is due to its effect on another upregulated atypical $\mathrm{PKC}, \mathrm{PKC} 2 / \lambda$ (Tsokas et al, 2016). Regardless of the specific aPKC ZIP disrupts, there is a consensus that this drug is a unique tool to study the maintenance of LTP. In our experiments, we used ZIP as a molecular tool to disrupt cocaine-evoked plasticity and evaluate if the hierarchical pattern of plastic changes between the VTA and NAc relies on ZIP-sensitive mechanism.

It is known that a single cocaine exposure is sufficient to induce LTP in the VTA (Ungless et al, 2001). Here, a single in vivo ZIP microinfusion into the VTA proved sufficient to block this cocaine-elicited enhancement of AMPA/NMDA ratio. Hence, ZIP microinfusion in the next set of experiments continuously and effectively blocked cocaine-evoked increase in AMPA/NMDA ratios. Daily ZIP administration into the VTA prevented an AMPA/NMDA ratio increase in putative DA cells of the VTA, as expected (Ho et al, 2012; VélezHernández et al, 2013). Interestingly, NAc core putative MSN also presented a disruption of plasticity. Unlike the VTA, one cocaine injection does not elicit plastic changes in the NAc (Thomas et al, 2001; Kourrich et al, 2007). It has been shown that repeated cocaine exposure causes an initial depression of synaptic strength and neuronal excitability in the NAc (Zhang et al, 1998; Thomas et al, 2001; Hu et al, 2004; Dong et al, 2006; Kourrich et al, 2007; Mameli et al, 2009;). In our hands, this reduction in synaptic strength was not present in the NAc core of animals treated with ZIP in the VTA. This suggests that the presence of a persistent increase in AMPA/NMDA ratio in the VTA participates in the decrease of AMPA/ NMDA ratio in the NAc core after cocaine administration.

Synaptic potentiation in the VTA can persist for several days after the last cocaine exposure (Ungless et al, 2001; Borgland et al, 2004). In our study, ZIP-treated animals did not present an increased synaptic strength in the VTA after a withdrawal period. Therefore, the disruption of cocaine-evoked increase in AMPA/NMDA ratio in the VTA can be longlasting. In addition, in the NAc it has been demonstrated that a withdrawal period enables a transition from a depressed state to increased synaptic potentiation, together with an elevated 
AMPA receptor surface expression (Boudreau and Wolf, 2005; Boudreau et al, 2007; Kourrich et al, 2007;Conrad et al, 2008). Our results do not show this enhanced synaptic potentiation after ZIP treatment. Together, this reveals that NAc core neuroadaptations, both in the forms of depressed and increased AMPA/NMDA ratio, seem to be mediated in part by the occurrence of a persistent increase in AMPA/NMDA ratio in the VTA.

Investigation indicates that a single cocaine exposure can elicit a maximally potentiated LTP in VTA DA cells (Ungless et al, 2001; Borgland et al, 2004;). Here we observed that 24 hours after a cocaine challenge, all groups presented an increase in synaptic strength, reflecting cocaine's acute effects. Similar to previous findings (Shema et al, 2009), the increase in synaptic potentiation in ZIP treated animals implies that this drug does not preclude the formation of new synaptic potentiation in the VTA. It has been shown that a cocaine challenge reverts synaptic potentiation and triggers AMPA receptor internalization in the NAc (Thomas et al, 2001; Boudreau et al, 2007; Kourrich et al, 2007). This effect was not observed in ZIP-treated animals, probably because they did not have an increased AMPA/NMDA ratio after the withdrawal period. Previous findings show that one single cocaine exposure does not increase AMPA receptor expression in the NAc, which could also explain the absence of any alterations in AMPA/NMDA ratio at this time point (Boudreau and Wolf, 2005).

Taken together our electrophysiological results reveal that NAc core neuroadaptations during cocaine sensitization, both in the forms of depressed and increased synaptic strength, are dependent on the persistence of an aPKC-mediated persistent increase in AMPA/NMDA ratio in the VTA.

We also evaluated how inhibition of cocaine-evoked enhancement of AMPA/NMDA ratio affects the expression of cocaine related behaviors in the animals used in the previous experiments. We observed that daily inhibition of synaptic potentiation in the VTA attenuates the initiation of sensitization. The results parallel previous findings from our laboratory, in which a single ZIP micronfusion disrupted cocaine sensitization (VélezHernández et al, 2013). Similar to our experiment, others have shown that daily ZIP microinfusions into the ventricle of mice, which reached several brain structures, progressively blocked cocaine sensitization (Howell et al, 2014). However, our study provides evidence that ZIP-induced behavioral effects are specifically due to an inhibition of AMPA/NMDA ratio increase in the VTA.

The expression of cocaine sensitization remained unaffected by the persistent inhibition of synaptic potentiation in the VTA. This suggests that NAc neuroadaptations mediating the maintenance of sensitization can develop independently of VTA LTP. There is a consensus that the expression of cocaine sensitization is mediated by the NAc (Kalivas, 1995; Wang et al, 1997). Previous experiments have shown that disruption of NAc plasticity by inhibition of AMPA receptors and aPKCs, block the maintenance of sensitization and other cocaine conditioned reward memories (Kalivas and McFarland, 2003; Li et al, 2011; Shabashov et al, 2011; Vélez-Hernández et al, 2013). Therefore, NAc neuroplasticity seems necessary for the expression of cocaine-evoked behaviors. Unlike these experiments, in our examination of the effect of ZIP in VTA, our subjects were not microinfused with this plasticity disruptor 
into the NAc. Hence, the expression of cocaine behavioral sensitization could have been mediated by direct cocainemediated plasticity changes in the NAc or other cellular processes that allow for the expression of sensitization (Borgland et al, 2006; Valjent et al, 2006). An alternative explanation could be that plasticity changes evoked by afferents from other brain areas unaffected by ZIP administration are the ones responsible for the maintenance of behavioral sensitization (Goto and Grace, 2005).

It did not escape our attention that some of the behavioral results observed did not reflect our electrophysiological findings. Considering that daily ZIP administration into the VTA only attenuated behavioral sensitization, this result adds to the literature indicating that there might be a temporal discrepancy between drug-adaptive behaviors and drug-evoked plasticity (Luscher \& Malenka 2011; Luscher et al 2013; Zhang et al 2016). For example, it was demonstrated that locomotor sensitization precedes the reduction of GABA inhibition in dopaminergic neurons, an event that leads to enhanced synaptic signaling (Liu et al, 2005). In our case, it could be that increases in VTA AMPA/NMDA ratio becomes more relevant for cocaine-related behaviors following consecutive drug exposures.

The unaltered expression of cocaine sensitization was surprising considering the disruption of NAc core synaptic plasticity following daily ZIP microinfusions into the VTA. This could imply that cocaine elicited behaviors can develop independently of some NAc core synaptic potentiation. Another explanation for the observed maintenance of sensitization is that synaptic enhancement of non-VTA synapses in the NAc could mediate the expression of cocaine sensitization. Previous research shows that ZIP microinfusions into the NAc reverts cocaine behaviors (Li et al, 2011; Shabashov et al, 2011; Vélez-Hernández et al, 2013). In our experiments ZIP was microinfused in the VTA, leaving non-VTA synapses unaltered. It could be presumed that non-VTA synapses in the NAc play a more vital role in the maintenance of cocaine sensitization such as the connection between the prefrontal cortex and NAc core (Pierce et al., 1998).

Finally, in our behavioral experiments, ZIP mediated changes in locomotion were due to a specific reduction in stereotypic movement. During locomotor sensitization, rats transition from ambulatory movement to an enhanced repetitive behavior, termed stereotypy (Wood et al, 1998), which includes intensive head bobbing, sniffing, and teeth clenching (Rebec, 2006). This type of behavior has been associated with the development of addiction (Henry and White, 1995; McClung and Hirsh, 1998). Therefore, ZIP-mediated disruption of this type of movement in the VTA indicates that stereotypy might rely on a cellular memory component.

In sum, our study proposes aPKC-mediated synaptic potentiation to be one of the "key signals" that elicits sequential plasticity formation between the VTA and the NAc during behavioral cocaine sensitization. Furthermore, the susceptibility of this potentiation to ZIP implies that this VTA-NAc sequential neuroadaptive signal relies on aPKC's, possibly PKM- $\zeta$ (Sacktor and Hell, 2017; Tsokas et al, 2016). Future experiments will require the use of contingent behavioral models to better assess the role of LTP in drug-associated behaviors. Having a better understanding of the cellular substrates that allow addiction 
memory formation, such as the aPKC isoforms, can support their exploration and relationship to drug addiction susceptibility in humans.

\section{Acknowledgments}

We want to extend our appreciation to Garrett Seale for the helpful editorial comments. Authors would also like to thank Karl Bosque-Cordero, Aynette Ramos-Cardona, Tara Ortiz-Ithier, Alan Montiel-Ramos, Luis Aponte-Cofresí, Tania M. Dominguez, Rebecca M. Parodi-Rullán, and Luis F. Centeno-Matos for their help with the neurosurgical procedures. We also thank Dr. Priscila Sanabria for her help with figure preparation and for feedback on the manuscript. This research was part of the student's dissertation requirement to obtain an M.D. /Ph.D. degree from the University of Puerto Rico, Medical Sciences Campus.

Funding and Disclosure

This work was supported by grants from National Institute of General Medical Sciences (2SC1GM084854-05A1) and National Science Foundation Partnerships for International Research and Education (OISE-1545803) to CAJR, Institutional National Institute on Minority Health and Health Disparities Research Centers in Minority Institutions Program (8G12MD007600), National Institutes of Health grants (2R37MH057068, MH115304 to T. Sacktor). Student support was provided by the National Center for Research Resources Research Initiative for Scientific Enhancement (R25 GM061838) to AVA, BSV, National Institute of General Medical Sciences (R25 NS080687) to AMR and National Center for Research Resources grant (2G12-RR003051) to CMR. Authors declare no conflicts of interest.

\section{References}

Bliss TV, Lomo T (1973). Long-lasting potentiation of synaptic transmission in the dentate area of the anaesthetized rabbit following stimulation of the perforant path. J Physiol (Lond) 232: 331-356. [PubMed: 4727084]

Borgland SL, Malenka RC, Bonci A (2004). Acute and Chronic Cocaine-Induced Potentiation of Synaptic Strength in the Ventral Tegmental Area: Electrophysiological and Behavioral Correlates in Individual Rats. Journal of Neuroscience 24: 7482-7490. [PubMed: 15329395]

Borgland SL, Taha SA, Sarti F, Fields HL, Bonci A (2006). Orexin A in the VTA Is Critical for the Induction of Synaptic Plasticity and Behavioral Sensitization to Cocaine. Neuron 49: 589-601. [PubMed: 16476667]

Boudreau AC, Reimers JM, Milovanovic M, Wolf ME (2007). Cell Surface AMPA Receptors in the Rat Nucleus Accumbens Increase during Cocaine Withdrawal But Internalize after Cocaine Challenge in Association with Altered Activation of MitogenActivated Protein Kinases. Journal of Neuroscience 27: 10621-10635. [PubMed: 17898233]

Boudreau AC, Wolf ME (2005). Behavioral Sensitization to Cocaine Is Associated with Increased AMPA Receptor Surface Expression in the Nucleus Accumbens. Journal of Neuroscience 25: 91449151. [PubMed: 16207873]

Conrad KL, Tseng KY, Uejima JL, Reimers JM, Heng L-J, Shaham Y, et al. (2008). Formation of accumbens GluR2-lacking AMPA receptors mediates incubation of cocaine craving. Nature 454: 118-121. [PubMed: 18500330]

Di Chiara G, Imperato A (1988). Drugs abused by humans preferentially increase synaptic dopamine concentrations in the mesolimbic system of freely moving rats. Proc Natl Acad Sci USA 85: 52745278. [PubMed: 2899326]

Dong Y, Green T, Saal D, Marie H, Neve R, Nestler EJ, et al. (2006). CREB modulates excitability of nucleus accumbens neurons. Nat Neurosci 9: 475-477. [PubMed: 16520736]

Engblom D, Bilbao A, Sanchis-Segura C, Dahan L, Perreau-Lenz S, Balland B, et al. (2008). Glutamate Receptors on Dopamine Neurons Control the Persistence of Cocaine Seeking. Neuron 59: 497-508. [PubMed: 18701074]

Everitt BJ, Wolf ME (2002). Psychomotor stimulant addiction: a neural systems perspective. J Neurosci 22: 3312-3320. [PubMed: 11978805] 
Ferrario CV, Li X, Wang X, Reimers JM, Uejima JL, Wolf ME (2010). The Role of Glutamate Receptor Redistribution in Locomotor Sensitization to Cocaine. Neuropsychopharmacology 35: 818-833. [PubMed: 19924109]

Goto Y, Grace AA (2008). Limbic and cortical information processing in the nucleus accumbens.Trends Neurosci. 31:552-8. [PubMed: 18786735]

Goto Y, Grace AA (2005) Dopamine-dependent interactions between lim- bic and prefrontal cortical plasticity in the nucleus accumbens: disruption by cocaine sensitization. Neuron 47:255-266. [PubMed: 16039567]

Henry DJ, White FJ (1995). The persistence of behavioral sensitization to cocaine parallels enhanced inhibition of nucleus accumbens neurons. Journal of Neuroscience 15: 6287-6299. [PubMed: 7666211]

Ho SY, Chen CH, Liu TH, Chang HF, Liou JC (2012). Protein Kinase MS Is Necessary for CocaineInduced Synaptic Potentiation in the Ventral Tegmental Area. BPS 71: 706-713.

Hollander JA, Carelli RM (2007). Cocaine-associated stimuli increase cocaine seeking and activate accumbens core neurons after abstinence. Journal of Neuroscience 27:3535-9. [PubMed: 17392469]

Howell KK, Monk BR, Carmack SA, Mrowczynski O, Clark R, Anangostaras S (2014). Inhibition of PKC disrupts addiction-related memory. Frontiers in Behavioral Neuroscience 8: 70 doi:10.3389/ fnbeh.2014.00070/abstract. [PubMed: 24639635]

Hu XT, Basu S, White F (2004). Repeated Cocaine Administration Suppresses HVACa2+ Potentials and Enhances Activity of K+ Channels in Rat Nucleus Accumbens Neurons. Journal of Neurophysiology 92: 1597-1607. [PubMed: 15331648]

Hyman SE, Malenka RC, Nestler EJ (2006). Neural mechanisms of addiction: the role of rewardrelated learning and memory. Annu Rev Neurosci 29: 565-598. [PubMed: 16776597]

Kalivas PW (1995). Interactions between dopamine and excitatory amino acids in behavioral sensitization to psychostimulants. Drug Alcohol Depend 37: 95-100. [PubMed: 7758408]

Kalivas PW, Duffy P (1993). Time course of extracellular dopamine and behavioral sensitization to cocaine. I. Dopamine axon terminals. Journal of Neuroscience 13: 266-275. [PubMed: 8423473]

Kalivas PW, McFarland K (2003). Brain circuitry and the reinstatement of cocaineseeking behavior. Psychopharmacology 168: 44-56. [PubMed: 12652346]

Kalivas PW, Stewart J (1991). Dopamine transmission in the initiation and expression of drug- and stress-induced sensitization of motor activity. Brain Res Brain Res Rev 16: 223-244. [PubMed: 1665095]

Kourrich S, Rothwell PE, Klug JR, Thomas MJ (2007). Cocaine Experience Controls Bidirectional Synaptic Plasticity in the Nucleus Accumbens. Journal of Neuroscience 27: 7921-7928. [PubMed: 17652583]

Lee AM, Kanter BR, Wang D, Lim JP, Zou ME, Qiu C, et al. (2013). Prkcz null mice show normal learning and memory. Nature 493: 416-419. [PubMed: 23283171]

Li YQ, Xue YX, He YY, Li FQ, Xue LF, Xu CM, et al. (2011). Inhibition of PKM in Nucleus Accumbens Core Abolishes Long-Term Drug Reward Memory. Journal of Neuroscience 31: 5436-5446. [PubMed: 21471379]

Ling DSF, Benardo LS, Serrano PA, Blace N, Kelly MT, Crary JF, et al. (2002). Protein kinase M $\zeta$ is necessary and sufficient for LTP maintenance. Nat Neurosci 5: 295-296. [PubMed: 11914719]

Liu Q, Pu L, Poo M (2005). Repeated cocaine exposure in vivo facilitates LTP induction in midbrain dopamine neurons. Nature 437: 1027-1031 [PubMed: 16222299]

Lu W, Man H, Ju W, Trimble WS, MacDonald JF, Wang YT (2001). Activation of synaptic NMDA receptors induces membrane insertion of new AMPA receptors and LTP in cultured hippocampal neurons. Neuron 29: 243-254. [PubMed: 11182095]

Lynch MA (2004). Long-term potentiation and memory. Physiological reviews 84: 87-136 [PubMed: 14715912]

Mameli M, Halbout B, Creton C, Engblom D, Parkitna JR, Spanagel R, et al. (2009). Cocaine-evoked synaptic plasticity: persistence in the VTA triggers adaptations in the NAc. Nature Publishing Group 12: 1036-1041. 
McClung C, Hirsh J (1998). Stereotypic behavioral responses to free-base cocaine and the development of behavioral sensitization in Drosophila. Curr Biol 8: 109-112. [PubMed: 9427649]

Owesson-White CA, Ariansen J, Stuber GD, Cleaveland NA, Cheer JF, Wightman RM, Carelli RM (2009). Neural encoding of cocaine-seeking behavior is coincident with phasic dopamine release in the accumbens core and shell. Eur J Neurosci. 30:1117-27. [PubMed: 19735286]

Parsons RG, Davis M (2011). Temporary disruption of fear-potentiated startle following PKMS inhibition in the amygdala. Nat Neurosci 14: 295-296. [PubMed: 21258326]

Pastalkova E, Serrano PA, Pinkhasova D, Wallace E, Fenton AA, Sacktor TC (2006). Storage of Spatial Information by the Maintenance Mechanism of LTP. Science 313: 1141-1144. [PubMed: 16931766]

Paxinos G, Watson C. The rat brain in stereotaxic coordinates. San Diego: Academic Press; 1998

Pierce RC, Reeder DC, Hicks J, Morgan ZR, Kalivas PW (1998) Ibotenic acid lesions of the dorsal prefrontal cortex disrupt the expression of behavioral sensitization to cocaine. Neuroscience 82:1103-1114 [PubMed: 9466434]

Rebec GV (2006). Behavioral Electrophysiology of Psychostimulants. Neuropsychopharmacology 31: 2341-2348. [PubMed: 16855534]

Saal D, Dong Y, Bonci A, Malenka RC (2003). Drugs of abuse and stress trigger a common synaptic adaptation in dopamine neurons. Neuron 37: 577-582 [PubMed: 12597856]

Saunders BT, Richard JM, Margolis EB, Janak PH (2018). Dopamine neurons create Pavlovian conditioned stimuli with circuit-defined motivational properties. Nature Neuroscience 21:10721083. [PubMed: 30038277]

Saunders BT, Robinson TE (2012). The role of dopamine in the accumbens core in the expression of Pavlovian-conditioned responses. Eur J Neurosci. 36:2521-32. [PubMed: 22780554]

Serrano P, Yao Y, Sacktor TC (2005). Persistent Phosphorylation by Protein Kinase M Maintains LatePhase Long-Term Potentiation. Journal of Neuroscience 25: 1979-1984. [PubMed: 15728837]

Shabashov D, Shohami E, Yaka R (2011). Inactivation of PKMS in the NAc Shell Abolished CocaineConditioned Reward. J Mol Neurosci 47: 546-553. [PubMed: 22127928]

Shema R, Hazvi S, Sacktor TC, Dudai Y (2009). Boundary conditions for the maintenance of memory by PKM in neocortex. Learning \& Memory 16: 122-128. [PubMed: 19181618]

Shema R, Sacktor TC, Dudai Y (2007). Rapid Erasure of Long-Term Memory Associations in the Cortex by an Inhibitor of PKM. Science 317: 951-953. [PubMed: 17702943]

Tan KR, Brown M, Labouèbe G, Yvon C, Creton C, Fritschy J-M, et al. (2010). Neural bases for addictive properties of benzodiazepines. Nature 463: 769-774. [PubMed: 20148031]

Thomas MJ, Beurrier C, Bonci A, Malenka RC (2001). Long-term depression in the nucleus accumbens: a neural correlate of behavioral sensitization to cocaine. Nat Neurosci 4: 1217-1223. [PubMed: 11694884]

Tsokas P, Hsieh C, Yao Y, Lesburgueres E, Wallace EJC, Tcherepanov A, et al. (2016). Compensation for PKM $\zeta$ in long-term potentiation and spatial long-term memory in mutant mice. eLife 5:e14846. [PubMed: 27187150]

Ungless MA, Whistler JL, Malenka RC, Bonci A (2001). Single cocaine exposure in vivo induces long-term potentiation in dopamine neurons. Nature 411: 583-587. [PubMed: 11385572]

Valjent E, Corvol J-C, Trzaskos J, Girault J-A, Hervé D (2006). BMC Neuroscience. BMC Neurosci 7: 20-11. [PubMed: 16512905]

Vélez-Hernández ME, Vázquez-Torres R, Velasquez-Martinez MC, Jiménez L, Báez F, Sacktor TC, et al. (2013). Inhibition of Protein Kinase MS (PKM $\zeta$ ) in the Mesolimbic System Alters Cocaine Sensitization in Rats. Journal of Drug and Alcohol Research 2: 1-12.

Volk LJ, Bachman JL, Johnson R, Yu Y, Huganir RL (2013). PKM- $\zeta$ is not required for hippocampal synaptic plasticity, learning and memory. Nature 493: 420-423 [PubMed: 23283174]

Wang GJ, Volkow ND, Fowler JS, Logan J, Abumrad NN, Hitzemann RJ, et al. (1997). Dopamine D2 receptor availability in opiate-dependent subjects before and after naloxone-precipitated withdrawal. Neuropsychopharmacology 16: 174-182. [PubMed: 9015800] 
Wood RD, Tirelli E, Snyder KJ, Heyser CJ, LaRocca TM, Spear LP (1998). Evidence for behavioral sensitization to cocaine in preweanling rat pups. Psychopharmacology 138: 114-123. [PubMed: 9718280]

Zhang M, Gosnell BA, Kelley AE (1998). Intake of high-fat food is selectively enhanced by mu opioid receptor stimulation within the nucleus accumbens. J Pharmacol Exp Ther 285: 908-914.

[PubMed: 9580643] 


\section{Highlights}

1. Increased AMPA/NMDA is key in mesolimbic cocaine evoked sequential plasticity

2. aPKC's mediate cocaine-evoked synaptic potentiation in the VTA

3. Disruption of aPKC-mediated increase AMPA/NMDA decrease cocaineevoked behavior 

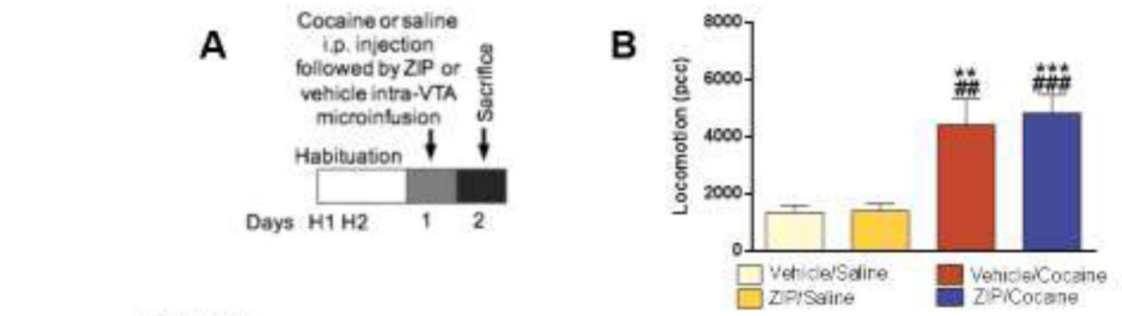

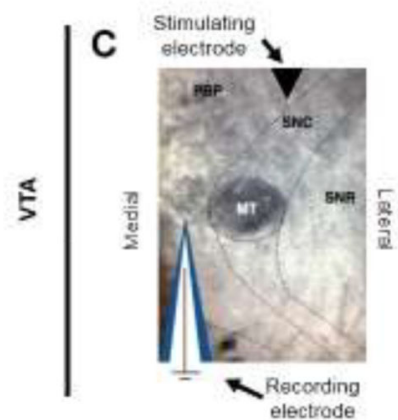

D
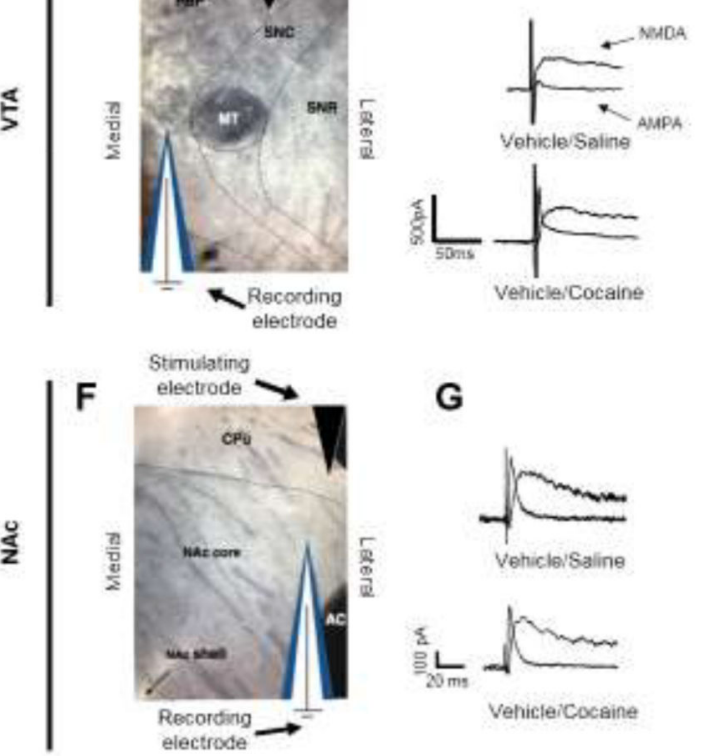

G
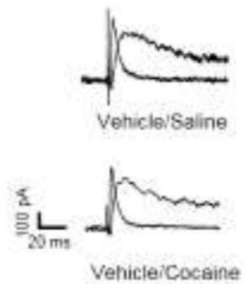

E

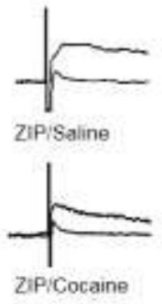

H
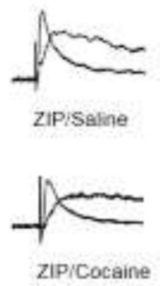
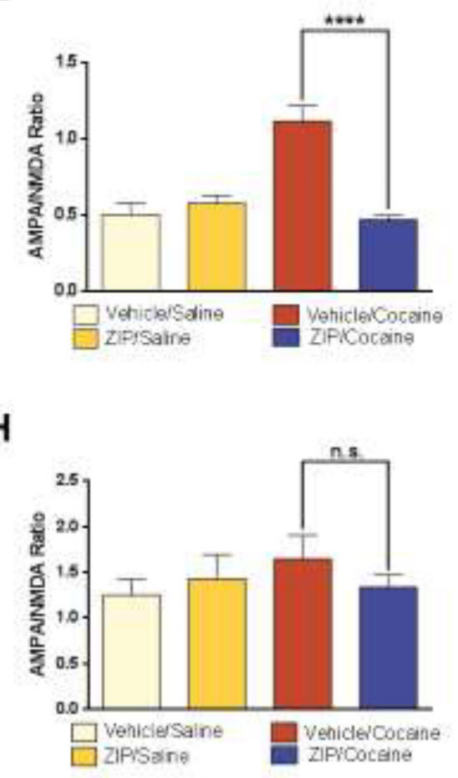

Figure 1. A single ZIP microinfusion into the VTA after acute cocaine exposure effectively disrupts VTA LTP

(A) Diagram of the behavioral sensitization and ZIP microinfusion protocol with the sacrifice and whole-cell recording time point. (B) Mean total locomotor activity (pcc/60min, \pm S.E.M.). Plus (+) and numeral (\#) signs denote group significance when compared to vehicle saline (*) and ZIP/Saline (\#) groups. (C) Image illustrating the placement of the recording electrode and the bipolar stimulating electrode in the VTA. (D) Sample traces display AMPA/NMDA ratio of VTA DA cells of all groups (scale: 200pA, 20ms). (E) Mean AMPA/NMDA ratios are presented in bar graphs (ratio + S.E.M.). a single ZIP microinfusions in the VTA of ZIP/Cocaine group blunted an AMPA/NMDA ratio increase of VTA DA cells when compared with Vehicle/Cocaine group (****, $p<0.0001$ Two-way ANOVA, followed by Sidak's multiple comparisons post-hoc test) (Vehicle/Saline: $n=6 / 6$, ZIP/Saline: $n=6 / 6$, Vehicle/Cocaine: $n=8 / 7$, ZIP/Cocaine: $n=6 / 6$ ). (F) Image illustrating the placement of the recording electrode and the bipolar stimulating electrode in the NAc Core. (G) Sample traces display AMPA/NMDA ratio of NAc core MSNs of all groups (scale: $100 \mathrm{pA}, 20 \mathrm{~ms})$. (H) In the NAc core, no significant difference was observed between all groups (Vehicle/Saline: $\mathrm{n}=6 / 6, \mathrm{ZIP} /$ Saline: $\mathrm{n}=6 / 6$, Vehicle/Cocaine: $\mathrm{n}=5 / 5$, ZIP/Cocaine: $\mathrm{n}=6 / 6)(\mathrm{p}>0.05$, Two-way ANOVA). $\mathrm{n}=$ number of cells $/$ number of animals. 

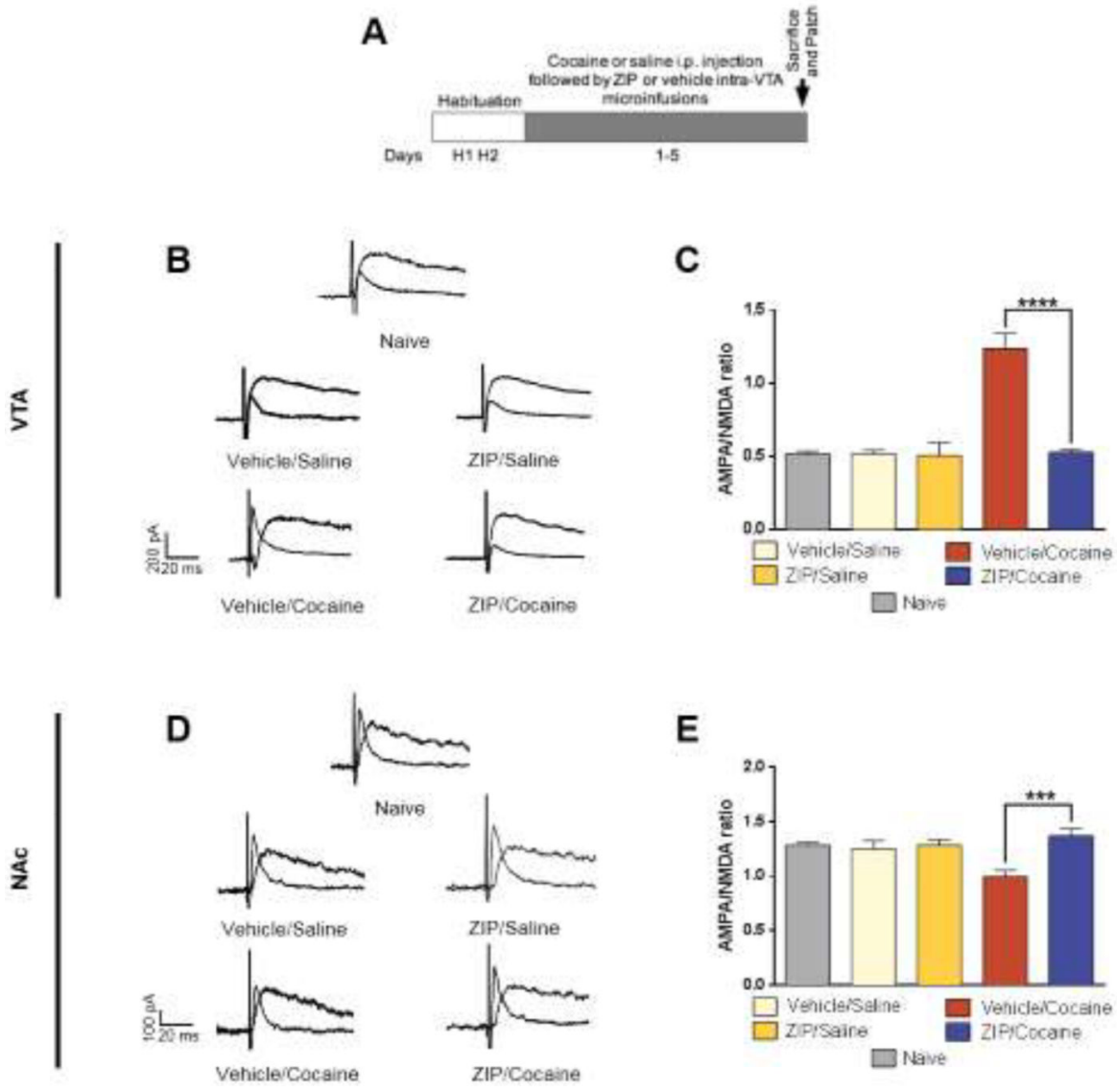

Figure 2. Daily ZIP microinfusions in the VTA blocks VTA LTP and disrupts NAc core plasticity by day 5 of the cocaine sensitization protocol.

(A) Diagram of the behavioral sensitization and ZIP microinfusion protocol with the sacrifice and whole-cell recording time point. (B) Sample traces display AMPA/NMDA ratio of VTA DA cells of naïve animals and all groups (scale: $200 \mathrm{pA}, 20 \mathrm{~ms}$ ). (C) Mean AMPA/NMDA ratios are presented in bar graphs (ratio \pm S.E.M.). Daily ZIP microinfusions in the VTA of ZIP/Cocaine group blunted an AMPA/NMDA ratio increase of VTA DA cells when compared with Vehicle/Cocaine group $(* * * *, \mathbf{p}<0.0001$, Two-way ANOVA, followed by Sidak's multiple comparisons post-hoc test) (Naïve: n6/6, Vehicle/Saline: $n=5 / 5$, ZIP/Saline: $n=6 / 6$, Vehicle/Cocaine: $n=8 / 6$, ZIP/Cocaine: $n=7 / 6$ ). (D) Image illustrating the placement of the recording electrode and the bipolar stimulating electrode in the NAc Core. (E) Sample traces display AMPA/NMDA ratio of NAc core MSNs of naïve animals and all groups (scale: $100 \mathrm{pA}, 20 \mathrm{~ms}$ ). (F) In the NAc core, a decrease in AMPA/NMDA ratio of ZIP/Cocaine group was not observed when compared with Vehicle/Cocaine group (***, p<0.001, Two-way ANOVA, followed by Sidak's multiple comparisons post-hoc test). No significant difference was observed in Naïve, Vehicle/Saline and ZIP/Saline control groups throughout the experiment (Naïve: n=8/7, Vehicle/Saline: $n=7 / 7$, ZIP/Saline: $n=6 / 6$, Vehicle/Cocaine: $n=17 / 9$, ZIP/Cocaine: $\mathbf{n = 1 8 / 9})(\mathrm{p}>0.05$, Two-way ANOVA). $\mathrm{n}=$ number of cells / number of animals. 
A
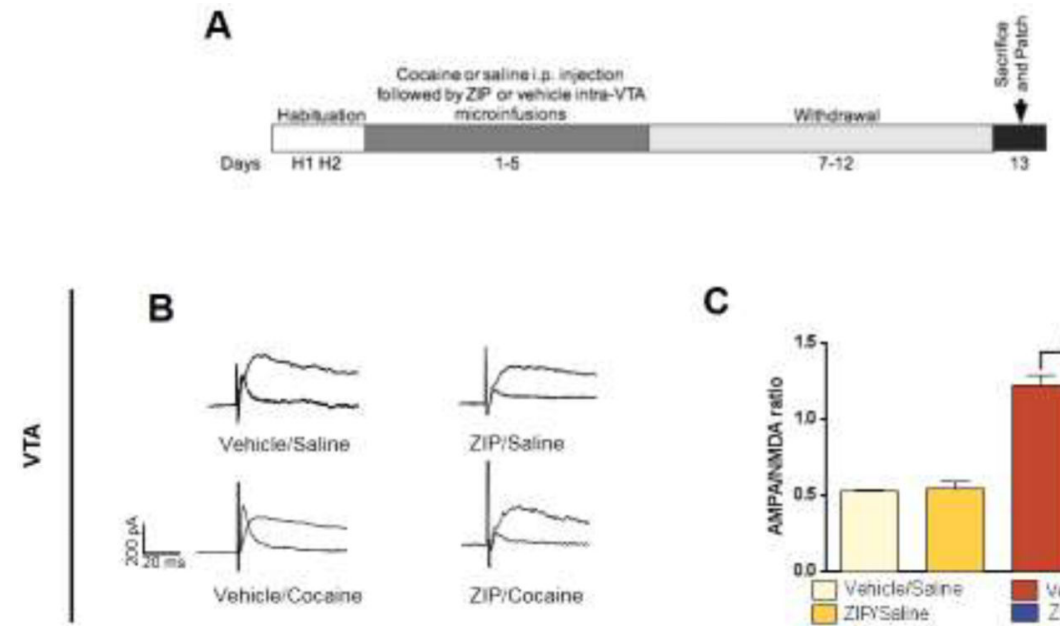

C
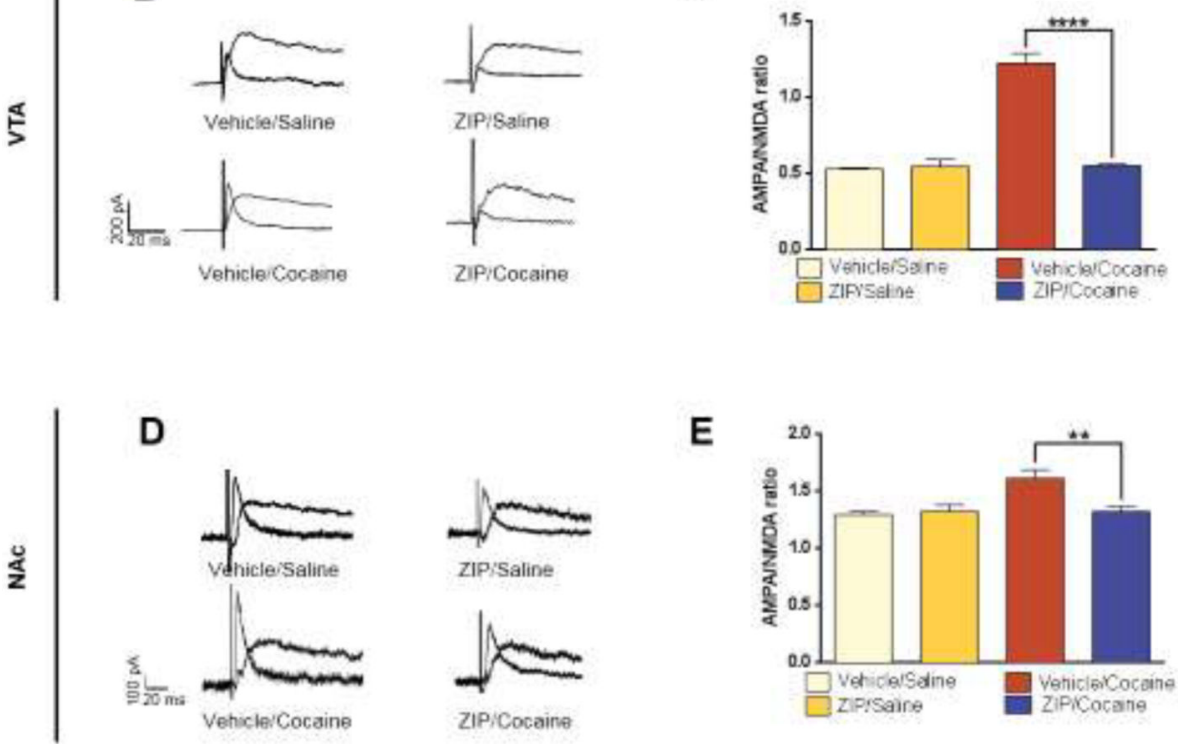

Figure 3. ZIP inhibition of VTA plasticity is long lasting and blunts NAc core synaptic potentiation after a withdrawal period.

(A) Illustration of the behavioral protocol and whole-cell recording time point. (B) Sample traces display AMPA/NMDA ratio of VTA DA cells of all groups (scale: $200 p A$, 20ms). (C) AMPA/NMDA ratios are presented in bar graphs (ratio \pm S.E.M.). After a withdrawal period, AMPA/NMDA ratio of $\mathrm{ZIP} / \mathrm{Cocaine}$ treated animals is significantly reduced when compared with Vehicle/Cocaine group (****, p<0.0001, Two-way ANOVA, followed by Sidak's multiple comparisons post-hoc test), and is not significantly different from control groups (Vehicle/Saline: $n=6 / 6$, ZIP/Saline: $n=7 / 6$, Vehicle/ Cocaine: $n=6 / 6$, ZIP/Cocaine: $n=9 / 7$ ). (D) Sample traces and (E) mean data display AMPA/NMDA ratio of NAc core MSNs of naïve animals and all groups (scale: 100pA, $20 \mathrm{~ms}$ ). After a withdrawal period, AMPA/NMDA ratios of NAc cells of ZIP/Cocaine animals do not show a significant increase as observed in the Vehicle/Cocaine group (**, p<0.001) (Vehicle/Saline: $\mathbf{n = 7 / 7}$, ZIP/Saline: $\mathbf{n = 5 / 5}$, Vehicle/Cocaine: $\mathbf{n = 1 1 / 8 , Z I P /}$ Cocaine: $\mathbf{n = 1 4 / 8}$ ). No significant difference was observed in Naïve, Vehicle/Saline and ZIP/ Saline control groups throughout the experiment ( $\mathrm{p}>0.05$, Two-way ANOVA). $\mathrm{n}=$ number of cells / number of animals. 


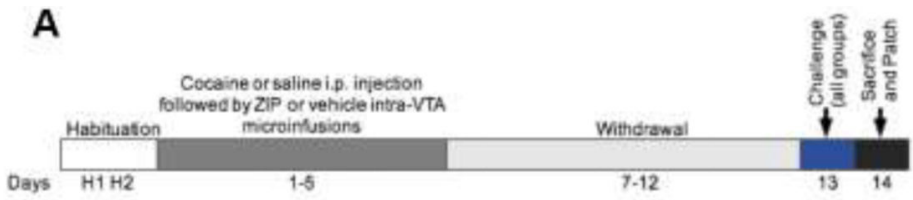

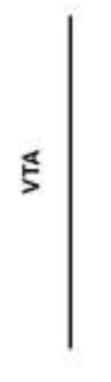

B
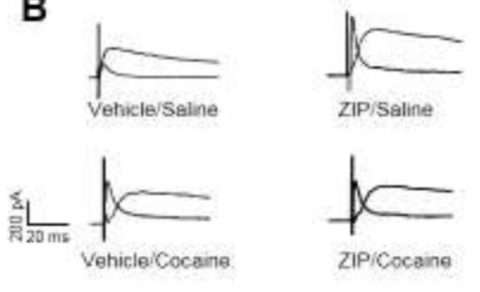

$\frac{\frac{9}{L}}{z}$

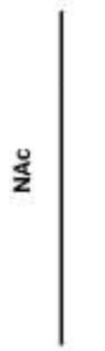

D
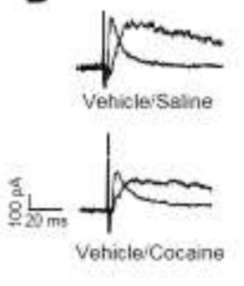

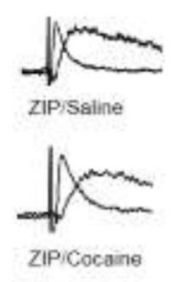

C

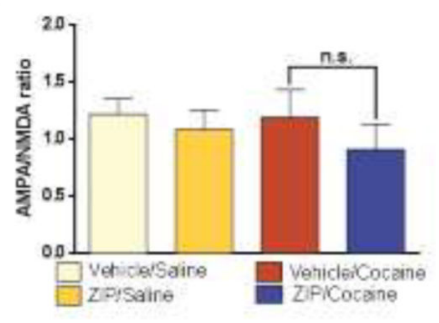

E

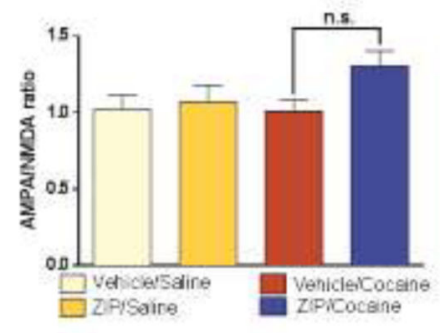

Figure 4. VTA plasticity can be generated again after ZIP inhibition and does not participate in NAc core plasticity after a cocaine challenge.

(A) Behavioral sensitization protocol and whole-cell recording time point. (B) Sample traces display AMPA/NMDA ratios of VTA DA cells of all groups (scale: 200pA, 20ms). (C) AMPA/NMDA ratios were presented in bar graphs (ratio \pm S.E.M.). After a cocaine challenge, Vehicle/Saline, ZIP/Saline and ZIP/Cocaine groups present an increase in AMPA/NMDA ratios (see fig. 2D). There is no significant difference between all groups (p>0.05, Two-way ANOVA) (Vehicle/Saline: $n=6 / 6$, ZIP/Saline: n=8/6, Vehicle/Cocaine: $n=4 / 4$, ZIP/Cocaine: $n=5 / 5)$. (D, E) Sample traces and mean data display AMPA/NMDA ratios of NAc core MSNs of naïve animals and all experimental groups (scale: $100 \mathrm{pA}, 20 \mathrm{~ms}$ ). Following a cocaine challenge, there is no significant difference between the AMPA/NMDA ratios of the NAc core MSN of all groups (Vehicle/Saline: $\mathbf{n = 6 , Z I P / S a l i n e : ~}$ =7, Vehicle/Cocaine: $n=11$, ZIP/Cocaine: $\mathbf{n = 9})(\mathrm{p}>0.05$, Two-way ANOVA). $\mathrm{n}=$ number of cells / number of animals. 


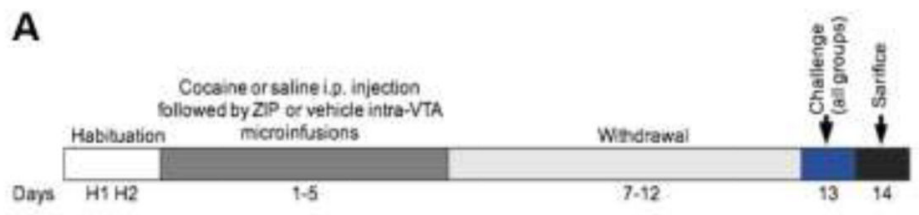

B
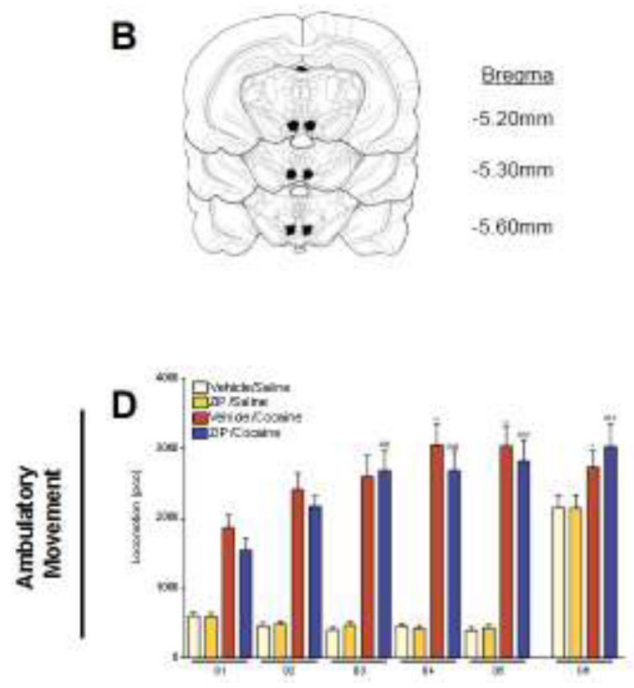
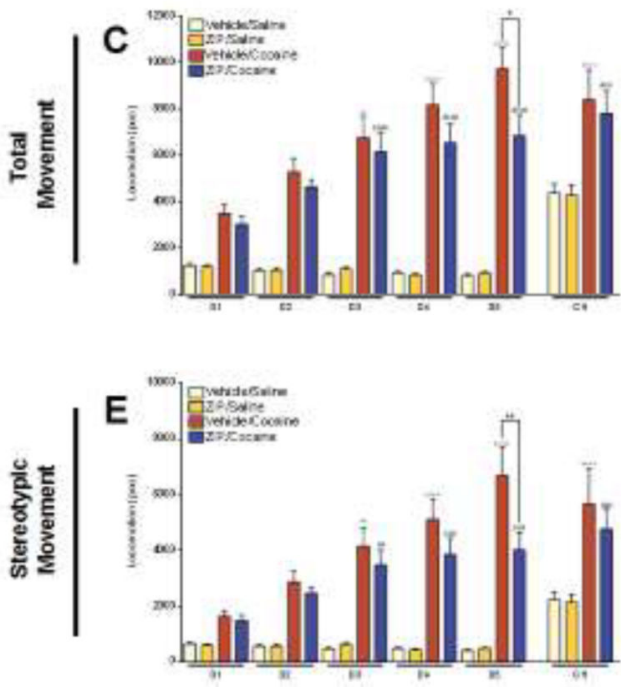

Figure 5. Daily ZIP microinfusions in the VTA attenuate cocaine sensitization. (A) The cocaine behavioral sensitization protocol. (B) Histological localization of infusion cannula within the VTA. (C, F, I) Mean total locomotor activity recorded each day for all groups (pcc/60min, \pm S.E.M.). Plus (+) and numeral (\#) signs denote significance in locomotor activity within the same group when compared to Day 1 (Two-way ANOVA, followed by Tukey's multiple comparisons post-hoc test). (C) Daily Intra-VTA ZIP microinfusions attenuated total locomotor sensitization of ZIP/Cocaine animals, evident by Day 5 (*, p=0.01, Two-way ANOVA, followed by Sidak's multiple comparisons post-hoc test). (D) No significant difference in ambulatory activity was observed (p>0.05, To-way ANOVA). (E) Stereotypic activity of ZIP/Cocaine animals was significantly reduced on day 5 when compared to Vehicle/Cocaine animals (**, $\mathbf{p = 0 . 0 0 1}$, Two-way ANOVA, followed by Sidak's multiple comparisons post-hoc test). No significant difference was observed in Vehicle/Saline and ZIP/Saline control groups throughout the experiment ( $\mathrm{p}>0.05$, Two-way ANOVA). (Vehicle/Saline n23/12, ZIP/ Saline n19/12, Vehicle/Cocaine 13/7, ZIP/Cocaine 20/14) $n=$ number of animals up to day $5 /$ number of animals on challenge day. Difference in numbers is due to use of these animals for the assessment of AMPA/NMDA ratios in Figures 1, 2 and 3. 